\title{
The New Academic Terminology: Adapting our Mission Statement and Terminology to Reflect the Contemporary Core Values of Academic Medical Centers
}

\author{
Martin Hauer-Jensen ${ }^{1 *}$, Keith R Laderoute ${ }^{2}$ and Mark H Phillips ${ }^{3}$ \\ ${ }^{1}$ Division of Radiation Health, University of Arkansas for Medical Sciences, Little Rock, AR, USA; \\ ${ }^{2} 810$ Coleman Avenue, Menlo Park, CA, USA; \\ ${ }^{3}$ Department of Radiation Oncology, University of Washington Medical Center, Seattle, WA, USA.
}

Submission: November 03, 2016; Published: December 13, 2016

*Corresponding author: Martin Hauer-Jensen, Division of Radiation Health, University of Arkansas for Medical Sciences, Little Rock, AR, USA, Tel: +1-501-686-7912, Fax: +1-501-421-0022; Email: mhjensen@life.uams.edu

\section{Introduction}

There has been a gradual shift in core values, focus and priorities at many academic medical centers around the world. The traditional core values that, in the past, justified the very existence of academic medical centers were, in popular terms,

a. To make sick people well (or at least better than they were before);

b. To train successors to take over as good doctors and scientists when the older generation retires; and

c. To generate new knowledge to help advance the field of biomedical science (euphemism for engaging in research).

Recently, most outsiders and many insiders have begun to look upon these idealistic core values as somewhat naïve (in fact, more than somewhat). Because it is also too complicated for many to have several different core values (what do you do when one core value conflicts with another?), the old core values and mission have recently been collapsed into a single one: the triple M mission, with "triple M" being short for Make More Money. While the triple $\mathrm{M}$ mission indeed reflects today's situation more accurately than the traditional core values, it does require rethinking of the old academic nomenclature, particularly in terms of how academic ranks and certain positions in the "system" are designated. Realizing that the current practice in this area is hopelessly behind the times, there is an urgent need to align the terminology with present-day goals.

We here propose the following update to describe individuals and strategies in academia:
A. RGZ (Revenue-Generating Zombie) - this is a replacement term for the current academic ranks of Professor, i.e., Assistant RGZ, Associate RGZ, and Full RGZ and so on. The RGZs are thought of as being the main contributors to and cornerstones of the triple $\mathrm{M}$ mission.

B. NMBT (No Money But Tenured). In contrast to the RGZ, the NMBT does not contribute significantly to the triple M mission. The vocalization for NMBT will likely be "numbbutt" (sits all day). A somewhat more flattering term will be devised for NMBTs that do remain academically productive.

C. NTCASE (Not Tenured, Covers All Salaries and Expenses). A subtype of RGZ with a particularly uncertain future. Again, the vocalization is interesting, in that it will sound like "nutcase".

D. $A A$ (Academic Apparatchik) -these are individuals who draw modest-to-large salaries, but rarely if ever generate something of value themselves. They should be regarded as extremely dangerous individuals. Most often, AAs justify their existence as bean counters, nay-sayers, drivers of the RGZ's, etc.

E. PENO (Public Enemy Number One) -when talking about multiple PENOs, it is critically important to pronounce this acronym distinctly to avoid confusion with other terms. These are individuals who are at the top or near the top of the academic pyramid. They are the powerful, but nameless, mostly unidentifiable institutional officials who epitomize the "corporate culture", somewhat along the lines of the Orwellian "Big Brother". The PENOs not only generate little or 
no value themselves, but, by virtue of their often demanding and belligerent attitude, frequently distract the RGZ's from their real goals, thereby causing them to actually generate less revenue than they would have done otherwise.

F. U-PENO (Associate/Vice PENO). These individuals would be consolidated as under-PENOs (or U-PENOs for short). Distinct vocalization remains critical for this term as well. The U-PENOs are the gophers of the PENOs. The RGZs and other individuals in the ranks could refer to the U-PENOs collectively as "scrotes". They are simply somewhat elevated AAs in terms of position, power, and salary. The threat against the RGZs posed by the PENOs is usually directly proportional to the size of the scrote-force.

G. $A K$ (Academic Kleptocrat). These are the most dangerous individuals in today's academia, the so-called "movers and shakers" of the academic medical center. The term denotes high-level administrators, "key institutional players", and other politically well connected individuals who consistently line their own pockets at the expense of RGZs. In other words, the AKs function according to the old principle of Soviet-style communism that "everything that you have is mine, and everything that I have is none of your business".

The new academic nomenclature presented here is obviously a work in progress (or regress?). It will be continuously updated and expanded and thus "move with the times". When a sufficient number of terms and acronyms have been gathered, an inspirational booklet for academic faculty could be published to keep them appropriately informed and remind them about why they are where they are.

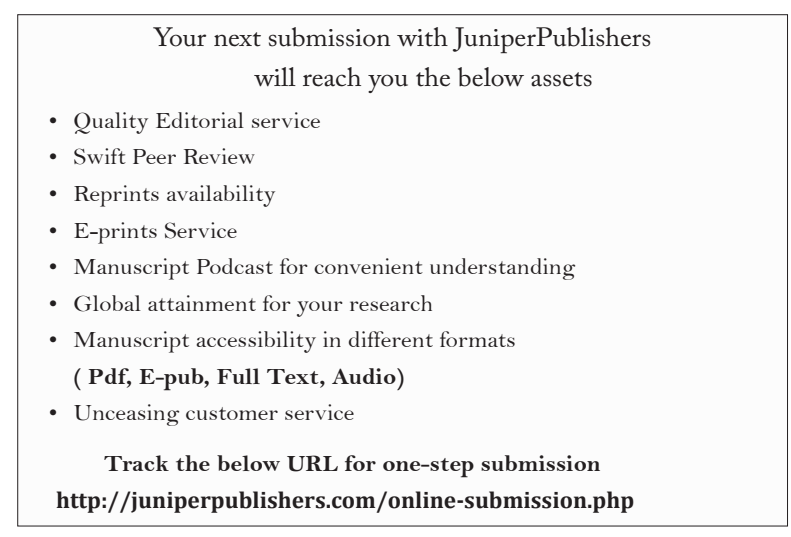

